\title{
On the maximal horizontal surface velocity for a rotational water wave near breaking
}

\author{
Calin Iulian Martin ${ }^{1}$
}

Received: 27 July 2015 / Accepted: 6 October 2015 / Published online: 17 October 2015

(C) Fondazione Annali di Matematica Pura ed Applicata and Springer-Verlag Berlin Heidelberg 2015

\begin{abstract}
We show that the maximum of the horizontal fluid velocity in a rotational deepwater plunging or spilling breaker is attained on the surface. Moreover, the mentioned maximum is, as a function of the time, almost everywhere differentiable, and its derivative is related to the horizontal component of the pressure gradient.
\end{abstract}

Keywords Rotational surface gravity waves - Time evolution · Hopf's maximum principle · Absolutely continuous function

Mathematics Subject Classification Primary: 35Q31, 35Q35, 76B15, 35R35 .

Secondary: 35B50

\section{Introduction}

This paper pertains to a very common feature of surface waves over deepwater regions, namely the tendency of these waves to turn over on themselves, situation that is the forerunner of wave breaking. The process of wave breaking has remained to a great extent theoretically unexplored in spite of considerable efforts over more than one hundred years of study.

Although very difficult — due to a variety of reasons, ranging from the nonlinearity of the equations and the boundary conditions to the instability of the measurement instruments carried away by the large ocean waves - the study of breaking waves is very important, since, cf. [1], breaking waves transfer horizontal momentum to surface currents, contribute to the mixing of uppers layers of the ocean, transport sediment in shallow water and intensify air-sea exchange of gases.

To cope with the inherent difficulties posed by breaking waves, several simplifying assumptions about the flow have to be made. They concern the neglect of the fluid viscosity,

Calin Iulian Martin

calin.martin@univie.ac.at

1 Fakultät für Mathematik, Universität Wien, Oskar-Morgenstern-Platz 1, 1090 Vienna, Austria 
of the surface tension (for fluid surfaces that are not highly curved). One more common simplification is the absence of vorticity in the fluid flow, which is a good approximation, except for the situation where there is a strong current shear. With respect to the latter assumption, we will allow for a rotational flow with constant vorticity. It is worth to point out that constant nonzero vorticity provides a good description of the regular tidal currents, cf. [4]. Also, for non-uniform currents the existence of a nonzero mean vorticity is more important than its specific distribution, cf. [10].

\section{Preliminaries}

We consider here a two-dimensional water flow, moving under the influence of gravity, such that the free surface waves propagate in the positive $x$-direction, while the $y$ axis points vertically upwards. The origin is assumed to be on the mean surface level. We assume the motion to be periodic in the $x$-direction with wavelength $L$. However, we do not require periodicity in the time variable $t$. The water flow occupies the domain $\Omega(t)$ bounded below by the flat bed

$$
y=-d,
$$

with $d>0$, and above by the free surface, which at any fixed time $t$ is described parametrically as

$$
x=\alpha(s, t), \quad y=\beta(s, t),
$$

with $\alpha$ and $\beta$ smooth function satisfying

$$
\begin{aligned}
& \alpha(s+L, t)=\alpha(s, t)+L \\
& \beta(s+L, t)=\beta(s, t)
\end{aligned} \quad \text { for all } s \in \mathbb{R}, t \geq 0,
$$

and, to cast out the singular points, we ask that

$$
\alpha_{s}^{2}(s, t)+\beta_{s}^{2}(s, t)>0 \text { for all } s \in[0, L] .
$$

If $P$ denotes the pressure, $\rho$ the (constant) density and $g$ the gravitational constant of acceleration, then the water flow with fluid velocity $(u, v)$ moves according to the Euler's equations

$$
\begin{aligned}
& u_{t}+u u_{x}+v u_{y}=-\frac{1}{\rho} P_{x}, \\
& v_{t}+u v_{x}+v v_{y}=-\frac{\rho}{\rho} P_{y}-g,
\end{aligned}
$$

the equation of mass conservation

$$
u_{x}+v_{y}=0
$$

and is subjected to the constraint of a positive constant vorticity $\gamma>0$; thus, it also fulfills the equation

$$
u_{y}-v_{x}=\gamma .
$$

Remark 2.1 There is a significant difference between positive and negative vorticity. For example, in certain geophysical contexts, the wind-induced current has constant vorticity, always of one sign (see, e.g., the equatorial undercurrent, cf. [5]). On the other hand, while the positive vorticity case $\gamma>0$ is suitable for the ebb current, negative vorticity is appropriate for the flood current, cf. [4,11]. 
From the point of view of mathematical analysis, in terms of the stream function $\psi$, defined up to a constant by $\psi_{y}=u$ and $\psi_{x}=-v$, the vorticity is expressed by means of the Poisson equation $\Delta \psi=\gamma$ and maximum/minimum principles depend upon the sign of the constant $\gamma$, cf. $[8,9]$.

Note that if the flow possesses the feature of having constant vorticity initially at time $t=0$, then the fact that the vorticity of a particle is preserved as the particle moves in a twodimensional flow (see [3]) ensures that this feature will persist at all later times. Equations of motion (2.2a)-(2.2c) are supplemented, cf. [6], by the dynamic boundary condition on the free surface

$$
P(\alpha(s, t), \beta(s, t), t)=P_{a t m},
$$

stating that the motion of the water is decoupled from the motion of the air above, and the kinematic boundary conditions

$$
\begin{aligned}
& v=0 \text { on } \quad y=-d, \\
& u \beta_{s}-v \alpha_{s}+\alpha_{s} \beta_{t}-\beta_{s} \alpha_{t}=0 \text { on the free surface, }
\end{aligned}
$$

stating the impermeability of the bed, in the first of the latter equations, while the second one indicates that the fluid flow relative to the free boundary is tangential to it. Equivalently, the conditions in (2.4) state that once a particle is on the bed or on the free surface, it will remain confined to that location for all times.

To guarantee the well posedness of smooth solutions to the governing equations (2.2a)(2.4), we assume that the exterior normal derivative of the pressure $\frac{\partial P}{\partial n}$ is negative all along the free surface, that is, there is a negative constant $C<0$ such that

$$
\frac{\partial P}{\partial n} \leq C<0
$$

on the free surface, at initial time $t=0$. It was shown, [7], that under condition (2.5), the governing equations are well posed, and moreover, within the existence time, condition (2.5) will persist.

\section{The time evolution of the maximum of $u$ at the free surface}

We are concerned in this section with the time evolution of the maximum of the horizontal fluid velocity. To begin with, we set for all $t \geq 0$

$$
M(t):=\max _{(x, y) \in \Omega(t)}\{u(x, y, t)\} .
$$

We claim that at any fixed time $t$, the maximum $M(t)$ is achieved only along the free surface. To prove the claim, notice that at a fixed time $t$, the function $(x, y) \rightarrow u(x, y, t)$ is harmonic in $\Omega(t)$, due to (2.2b) and (2.2c). Thus, since $u$ is not constant [u constant would force $v(x, y, t)=-\gamma x+f(t)$ for some function $f(t)$, by (2.2b) and (2.2c), which is impossible by (2.4)], its maximum can only be assumed on the boundary. Admitting for a moment that the maximum is achieved on the bed at some point $\left(x_{0},-d\right)$ at some time $t_{0}$, we infer from Hopf's maximum principle that

$$
u_{y}\left(x_{0},-d, t_{0}\right)<0 \text {. }
$$


But then, from (2.2c) we see that $v_{x}=u_{y}-\gamma<0$, which is a contradiction with the kinematic boundary condition on the bed, thus proving the claim. Therefore,

$$
M(t)=\sup _{(x, y) \in \Omega(t)}\{u(x, y, t)\}=\sup _{s \in[0, L]}\{u(\alpha(s, t), \beta(s, t), t)\} \text { for all } t \in[0, T),
$$

where $T>0$ is the breaking time.

We will now sketch the proof of the following result, which relates $M(t)$ to the pressure gradient on the free surface.

Theorem 3.1 The function $t \rightarrow M(t)$ is absolutely continuous and therefore almost everywhere differentiable, with

$$
M^{\prime}(t)=-\frac{1}{\rho} P_{X}(X(t), t) \text { for almost all } t \in(0, T),
$$

where $X(t)=(x(t), y(t))$ denotes any location where $u$ attains its maximum.

We state first a technical result that will help in the proof of Theorem 3.1. Its proof follows the line of proof of formula (3.3) in [6] (see also the considerations in [2]) and will therefore be omitted.

Lemma 3.2 Let $\xi(t)$ be such that $X(t)=(\alpha(\xi(t), t), \beta(\xi(t), t))$. Then the function $t \rightarrow$ $M(t)$ is absolutely continuous with

$$
M^{\prime}(t)=u_{t}(X(t), t)+u_{x}(X(t), t) \alpha_{t}(\xi(t), t)+u_{y}(X(t), t) \beta_{t}(\xi(t), t)
$$

for almost all $t \in[0, T)$.

Proof of Theorem 3.1 Since $s=\xi(t)$ is the maximum of the function $s \rightarrow u(\alpha(s, t), \beta(s, t)$, $t$ ), we infer that

$$
u_{x}(X(t), t) \alpha_{s}(\xi(t), t)+u_{y}(X(t), t) \beta_{s}(\xi(t), t)=0 .
$$

Assuming that at the instant $t$ where $M$ is differentiable, we have that $\alpha_{s}(\xi(t), t) \neq 0$, we divide the latter equality by $\alpha_{s}(\xi(t), t)$ and multiply then the result by $\alpha_{t}(\xi(t), t)$, we obtain, using also the kinematic boundary condition on the surface, that

$$
\begin{aligned}
u_{x}(X(t), t) & \alpha_{t}(\xi(t), t)+u_{y}(X(t), t) \beta_{t}(\xi(t), t) \\
& =u_{y}(X(t), t)\left(\beta_{t}(\xi(t), t)-\frac{\beta_{s}(\xi(t), t)}{\alpha_{s}(\xi(t), t)} \alpha_{t}(\xi(t), t)\right) \\
& =u_{y}(X(t), t) \frac{v(X(t), t) \alpha_{s}(\xi(t), t)-u(X(t), t) \beta_{s}(\xi(t), t)}{\alpha_{s}(\xi(t), t)} \\
& =u_{y}(X(t), t) v(X(t), t)+u(X(t), t) u_{x}(X(t), t),
\end{aligned}
$$

where the last equality was inferred from (3.4). The assertion in Theorem 3.1 becomes evident if we take into account the latter equality (3.3) and the first equation from (2.2a).

\section{The time evolution of the minimum of $u$ in the irrotational case}

The setting in this part of the paper is that of an irrotational flow. Therefore, using the equation of mass conservation (2.2b) and (2.2c) for $\gamma=0$ we see that $u$ is harmonic; thus, at any fixed time $t$, the function $(x, y) \rightarrow u(x, y, t)$ achieves its minimum on the boundary of the fluid 
domain. Arguing as in [6] or as in the beginning of Sect. 3, we infer that the minimum of $u$ is achieved on the free surface. Thus,

$$
m(t):=\min _{(x, y) \in \Omega(t)}\{u(x, y, t)\}=\min _{s \in[0, L]}\{u(\alpha(s, t), \beta(s, t), t)\} \text { for all } t \in[0, T),
$$

where $T>0$ is the breaking time. As in the case of the maximum of $u$, there is a link between $m(t)$ and the pressure gradient, as follows.

Theorem 4.1 The function $t \rightarrow m(t)$ is absolutely continuous and therefore almost everywhere differentiable. Moreover, the following holds

$$
m^{\prime}(t)=-\frac{1}{\rho} P_{x}(\tilde{X}(t), t) \text { for almost all } t \in(0, T),
$$

where $\tilde{X}(t)=(\tilde{x}(t), \tilde{y}(t))$ is any position where $u$ attains its minimum.

The following result concerning the precise location of the minimum of the horizontal velocity $u$ is true.

Proposition 4.2 Provided symmetry persists, the minimal horizontal fluid velocity is attained at the wave trough.

Proof According to the discussion in Section 4 of the paper [6], the minimum of the pressure $P$ is attained all along the free surface and the derivative of $P$ in any direction that points outside the fluid, at the free surface, is negative. Since $P$ is atmospheric at the free surface, we infer from the first assertion that

$$
P_{x}(\alpha(s, t), \beta(s, t), t) \alpha_{s}(s, t)+P_{y}(\alpha(s, t), \beta(s, t), t) \beta_{s}(s, t)=0, \quad s \in \mathbb{R}, t \geq 0,
$$

while the second assertion is equivalent to

$$
-P_{x}(\alpha(s, t), \beta(s, t), t) \beta_{s}(s, t)+P_{y}(\alpha(s, t), \beta(s, t), t) \alpha_{s}(s, t)<0 \text { for } s \in \mathbb{R}, t \geq 0 .
$$

Consequently, from the previous two relations, we have that

$$
-P_{x}(\alpha(s, t), \beta(s, t), t) \cdot \frac{\alpha_{s}^{2}(s, t)+\beta_{s}^{2}(s, t)}{\beta_{s}}<0,
$$

at all points on the free surface, except for the wave crest and the wave trough.

Assuming now that the minimum horizontal fluid velocity is attained at some point $X_{+}(t)$ on the declining part of the free surface, the same value must be attained at the symmetric point $X_{-}(t)$ on the ascending part of the wave. But then, it is clear from (4.5) that $P_{x}\left(X_{-}(t), t\right)>0$ and $P_{x}\left(X_{+}(t), t\right)<0$, unless the point in question is at the wave crest or at the wave trough. The latter two inequalities are in contradiction, since, in formula (4.2), we are allowed to choose any point $\tilde{X}(t)$ where the minimum is attained. As a consequence, the minimum is either at the wave crest or at the wave trough. Since initially we start from a symmetric traveling wave, in which case the value of $u$ at the wave trough is smaller than the value of $u$ at the wave crest, we can conclude that the minimum of $u$ is located at the wave trough, where $P_{x}=0$.

Acknowledgments The author is grateful to the referee for the comments and suggestions that improved the paper. 


\section{References}

1. Cokelet, E.D.: Breaking waves. Nature 267, 769-774 (1977)

2. Constantin, A., Escher, J.: Wave breaking for nonlinear nonlocal shallow water equations. Acta Math. 181, 229-243 (1998)

3. Constantin, A.: Nonlinear Water waves with Applications to Wave-Current Interactions and Tsunamis, vol. 81 of CBMS-NSF Regional Conference Series in Applied Mathematics. Society for Industrial and Applied Mathematics (SIAM), Philadelphia, PA (2011)

4. Constantin, A., Kalimeris, K., Scherzer, O.: A penalization method for calculating the flow beneath traveling water waves of large amplitude. SIAM J. Appl. Math. 75(4), 1513-1535 (2015)

5. Constantin, A., Johnson, R.S.: The dynamics of waves interacting with the Equatorial Undercurrent. Geophys. Astrophys. Fluid Dyn. 109(4), 311-358 (2015)

6. Constantin, A.: The time evolution of the maximal horizontal surface fluid velocity for an irrotational wave approaching breaking. J. Fluid Mech. 768, 468-475 (2015)

7. Coutand, D., Shkoller, S.: Well-posedness of the free-surface incompressible Euler equations with or without surface tension. J. Am. Math. Soc. 20, 829-930 (2007)

8. Fraenkel, L.E.: An Introduction to Maximum Principles and Symmetry in Elliptic Problems. Cambridge University Press, Cambridge (2000)

9. Gilbarg, D., Trudinger, N.S.: Elliptic Partial Differential Equations of Second Order. Springer, Berlin (2001)

10. Teles da Silva, A.F., Peregrine, D.H.: Steep, steady surface waves on water of finite depth with constant vorticity. J. Fluid Mech. 195, 281-302 (1988)

11. Yanagi, T.: Coastal Oceanography. Kluwer, Dordrecht (2003) 